\title{
Coeliac disease: neurological symptoms and association with Multiple Sclerosis
}

DKD Smyth: Senior Lecturer, Birmingham City University, Edgbaston, West Midlands.

N Abel: Senior Lecturer, Birmingham City University, Edgbaston, West Midlands.

Contact email: dion.smyth@bcu.ac.uk

Key words:

- Coeliac disease

- Multiple Sclerosis

- Auto-immune diseases

\section{Key points}

- Coeliac disease and MS are common conditions and the cause of considerable morbidity, reduced quality of life and mortality

- There are similarities in the symptom experience, presentation and clinical course of both conditions

- Some studies suggests an association between coeliac disease and MS

- As our knowledge of immunology develops and the relationship between auto-immune diseases evolves, the MS nurses role will need to advance to better assess and address the needs of this particular client group

Coeliac disease (CD) and Multiple Sclerosis (MS) are common and complex auto-immune conditions associated with significant morbidity and mortality. This article will present a description of both conditions, the main neurological manifestations associated with CD and describe the putative relationship between the two conditions. 


\section{Coeliac disease}

Coeliac disease (CD), which is otherwise known as 'gluten-sensitive enteropathy', 'non-tropical sprue' or 'coeliac sprue', is a lifelong systemic auto-immune condition characterised by a state of abnormally amplified immunological responsiveness to ingested gluten and related proteins in genetically susceptible individuals (Husby et al, 2012).

The classical presentation of coeliac disease was considered to be chronic malabsorption, weight loss and steatorrhea, reflecting damage sustained by the proximal small bowel mucosa; however, the clinical presentation of the condition is now recognised to be much more heterogeneous and can vary from silent or mild symptoms to overt severe reactions that most commonly affect the gut but might also affect other organs (Goddard \& Gillett, 2006; Finsterer \& Leutmezer, 2014)

The aberrant immune reaction is induced by the digestion of dietary wheat gluten and similar storage proteins in barley and rye (see Figure 1), which are ubiquitous in the Western diet, where the viscous thickening and elastic properties of the proteins are used widely in the production of bread, pastes, pastas, noodles, biscuits and sauces to provide texture and taste (Tjon et al, 2010).

Excluding from the diet any and all gluten containing cereals and foodstuffs can result in swift and significant symptom relief (Murray et al, 2004); however, it is recognised that adhering to such a diet is demanding and difficult (Zarkadas et al, 2006).

Figure 1: Causative gluten proteins in coeliac disease

\begin{tabular}{|l|l|}
\hline \multicolumn{2}{|c|}{ Gluten trigger proteins } \\
\hline Wheat & Gliadins and glutenins \\
\hline Barley & Hordeins \\
\hline Rye & Secalins \\
\hline
\end{tabular}




\section{Multiple Sclerosis}

Multiple sclerosis is a chronic auto-immune disease of the brain and spinal cord, where auto-reactive lymphocytic cell activity is directed against the myelin and axons (Ward-Abel, 2008)). The disease is characterized by early periods of relapse and remission as initially short-lived inflammation and remyelination occurs; however, this capacity for repair does not endure as oligodendrocytes are depleted, and progressive demyelination and axonal neurodegeneration with attendant disability develops (Compston and Cole, 2008).

The exact trigger of this auto-immune disorder remain elusive; however, it is suggested that MS is most likely a result of a complex interplay of environmental risk factors and genetic susceptibility (Compston and Cole, 2008; Malik et al, 2014) (see figure).

Figure: examples of suggested environmental triggers for MS

\section{Environmental risk factors in MS}

Latitudinal gradient (increasing distance north or south of the equator) - also related to vitamin D deficiency, climatic factors \& migratory patterns

Vitamin D deficiency

Infection - such as Epstein-Barr virus

Smoking

\section{Epidemiology}

\section{Coeliac disease}

Although coeliac disease was once considered to be uncommon (Leeds et al. 2008), the evidence from serological screening studies suggests the prevalence of coeliac disease in healthy global populations is around 1\% (Rubio-Tapia et al, 2012). Whilst there are reports of some geographical variation in incidence and prevalence, such as fewer cases in Finland than Germany (Ludvigsson et al, 2015), overall the western world frequency seems comparable, and similar to the prevalence of 
the disease in the Middle East and Australasia. This figure is thought also most likely to be an underestimation of cases with many people living with but not currently identified as having the condition, suggesting the actual incidence worldwide will be higher (Reilly \& Green, 2012).

There have been fewer reports of coeliac disease described in oriental and sub-Saharan African populations (Kang et al, 2013), where it has been hypothesized that the predominantly rice based diet wields an environmental sparing effect in such regions (Ciclitira et al, 2010); however, some literature suggests that coeliac disease might not be so rare in places such as China, rather the diagnosis is missed (Jiang et al, 2009). As the diet of developing countries or countries with primarily rice based diet consumption becomes 'westernized' and the ingestion of wheat based products increases, the prevalence of coeliac disease is predicted to likely rise (Sapone et al, 2012).

The true figures and increase in incidence will be supplemented by the growth in cases found by enhanced diagnostic techniques and improved awareness of the disease, both of which have contributed to the growing prevalence (Ludvigsson et al, 2015). Nevertheless, missed diagnosis, as well as delays in establishing a diagnosis of the disease, is reported consistently in the literature and explained by issues such as atypical symptomatic presentations or a lack of clinician consciousness of the condition (Zipser et al, 2005; Jones and Sleet, 2009; Viera et al, 2011).

\section{Multiple Sclerosis}

MS is the foremost cause of neurological disability in young adults with over 2 million people living with the disease worldwide, though the lack of accurate global data might suggest this figure be considered to be an underestimate (Malik et al, 2014).

The incidence is highest in countries peopled by Northern Europeans and their progeny (Compston and Cole, 2008), with negligible incidence in some African states and low incidence in China and the far East (Bomprezzi \& Craig, 2009). Simpson et al (2011) presented evidence that the incidence of MS increases with increasing global latitude; they suggested that variations from the worldwide distributional gradient, which is seen in lower than expected incidence in northern Sardinia for example, may be attributed to genetic and behavioural-cultural factors.

In the UK, Mackenzie et al (2013) estimated that 126669 people were living with MS in 2010 (203.4 per 100000 population). The prevalence within the UK rises most strikingly the further north one ventures, with Orkney recording the highest occurrence worldwide (Visser et al, 2012).

\section{Immune mechanisms and genetics}


Coeliac disease is a chronic systemic immune mediated disease characterised by reversible inflammatory changes and flattening of the villi in the jejunal duodenal mucosa in genetically predisposed people (di Sabatino and Corazza, 2009). Whilst the exact mechanisms of the tissue destruction are yet to be explicated, as with many auto-immune disorders it is recognised that there are both innate and adaptive immune responses; it has been established that predisposition to CD is strongly associated with the HLA (Human Leucocyte Antigen) locus, specifically MHC (major histocompatibility class) II genes that encode HLA-DQ2 (HLA DQA1*0502/DQB1*0201) and HLA-DQ8 (HLA DQB1*0302), located on chromosome 6p21 (Sapone et al, 2012). The majority of patients with CD (95\%) express genes that encode HLA-DQ2; the remainder HLA-DQ8 (Sapone et al, 2012).

The ingestion of the environmental trigger, gluten peptides, in susceptible individuals during periods of increased gut permeability initiates HLA-DQ2- and HLA-DQ8-positive antigen-presenting cells, which subsequently activates the adaptive immune system CD4+ T-cell lymphocyte response inducing the release of pro-inflammatory cytokines, such as Interferon- $\curlyvee$ and Inter-leukin-15 (Pagliari et al, 2015). This triggers the cytotoxic activity of the intraepithelial t-lymphocytes, which leads ultimately to infiltration and proliferation of lymphocytes to the gut mucosa, chronic inflammation hyperplasia of the crypts, and subtotal or complete villous atrophic alteration of the lamina propria, the loose connective tissue layer beneath the epithelium of the gut (Williams et al, 2010; Pagliari et al, 2015).

Unlike classical $C D$, the nature of the exact mechanism in MS remains elusive. MS is most likely triggered by an intricate interaction between multiple genes and climatic, infectious and/or other environmental factors, which results in inflammatory-mediated central nervous system degradation, deterioration and disability as a result of auto-reactive CD4 T-helper cells activity against myelin selfantigens (Karussis,2014).

That MS might be associated with a genetic factors increasing susceptibility when gene activity is modified by environmental factors is suggested from the evidence from epidemiological studies of greater rates of prevalence of MS in discrete populations, and the augmented familial risk; but no single underlying gene for MS has been identified (Bomprezzi \& Craig, 2009). Nonetheless, as with $\mathrm{CD}$, the strongest genetic association relates to gene variants of the major-histocompatibility complex region, supporting the suggestion that MS has an auto-immune aetiology (Milo \& Miller, 2014); that notwithstanding, the identified HLA risk alleles for genetic predisposition to MS differ to those of CD (HLA-DRB1*1501 and DR2 haplotypes in the case of MS). Nonetheless, it has been suggested that a co-expression of different combinations of the gene variants is likely in the subgroup population of patients with both disorders (Mokarizadeh et al, 2015). 
Conventionally, MS was considered a T-cell dominated disorder; however, evidence suggests the more active participation of $\mathrm{B}$ cells in the pathogenesis of the disease, acting as antigen presenting cells, preparing and regulating T-cells and as regulatory and pro-inflammatory mediators (Pröbstel et al, 2015; von Budingen et al, 2015). Although the target antigen of B cells in MS have yet to be fully identified, it has been hypothesised that antibody against gliadin, may bind to and develop demyelination of neural antigens and promote penetration of the blood brain barrier (Mokarizadeh et al, 2015).

\title{
Coeliac disease and MS
}

A common quality of auto-immunity is the phenomenon of coincidental disease (Hall \& Yates, 2010). The increased co-occurrence and association of $C D$ and other autoimmune and extra-intestinal diseases has long been observed and reported (Kumar et al, 2001; Lauret \& Rodrigo, 2013) [see table], and estimated to be least 10 times more frequent in the population of patients with CD than the general population (Rodrigo et al, 2014). MS is similarly known to be associated with other autoimmune diseases (Batur-Caglayan et al, 2012). The causes for the onset and manifestation of CD associated diseases are diverse and often yet to be elucidated; however, it is recognised that some, such as the association with type 1 diabetes mellitus and auto-immune thyroid disorders share a similar genetic HLA basis of predisposition, namely the DQ2 allele (Kumar et al, 2001). CD and other auto-immune diseases may share similar pathogenic mechanisms, such as impaired intestinal permeability resulting in increased circulation of immune stimulating infectious agents, or luminal antigens such as gliadin causing dysregulation of the immune system, whereby aberrant intestinal $T$ -lymphocytes trigger tissue damage, and increased antibody-mediated interference of transglutaminase (TGt-2) results in the development and action of autoantibodies against substrate proteins (Lauret \& Rodrigo, 2013).

Table: Examples of coeliac disease and associated auto-immune diseases

\author{
Endocrine diseases \\ Diabetes Mellitus \\ Auto-immune thyroid disease \\ Addison's disease
}

Dermatological diseases 
Dermatitis herpetiformis

Vitiligo

Alopecia areata

Rheumatological/connective tissue diseases

Rheumatoid arthritis

Systemic lupus ertythmatosus

Sjorgen's syndrome

Cardiological diseases

Auto-immune pericarditis

Dilated cardiomyopathy

Liver diseases

Auto-immune hepatitis

Primary biliary cirrhosis

Primary sclerosing hepatitis

Though the relationship remains contentious (Nicoletti et al, 2008; ), in part due to methodological limitations of small population sizes leading to studies being statistically underpowered (Salvatore et al, 2014), an increased prevalence of CD in patients with Multiple Sclerosis, and their close relatives, compared to the general population has been reported in prospective observational studies (Rodrigo et al, 2011) and the association described in a number of case report studies (Ferro et al, 2008; Batur-Caglayan et al, 2012; Shaygannejad et al, 2013; Azizi et al, 2014).

In their study, Rodrigo et al (2011) found an increased incidence of TGt anti-IgA antibody serological indicators of CD in patients with Relapsing Remitting Multiple Sclerosis, and evidence of histological changes (duodenal lesions in $29 \%$ of the sample, and mild villous atrophy in $11.1 \%$ of the sample); there was no variance between the genetic markers (DQ-2 \& DQ8 alleles) between the control and patient populations. 


\section{Clinical features and presentation of coeliac disease and multiple sclerosis}

Coeliac disease can present at any age; previously it presented most commonly either very early in life (aged 9-24 months, after weaning on wheat based products) or later in life, during the third and fourth decades (Lebenthal, 2008). In the UK adult case presentation tends to be more frequent with a peak during the 4 th decade of life (Hopper et al, 2007).

Hadjilivassilou et al (2010) describe how the word 'coeliac' is derived from 'koiliaki', the Greek word for abdominal, and there are a wide range of gastro-intestinal clinical manifestations of the disease (see table 1). The classical presentation of gastro-intestinal symptoms meant that the disease was initially typically thought of as exclusively related to disorder of the gut. Nevertheless, it is now appreciated that atypical or silent presentation includes features not necessarily associated with the gastro-intestinal tract (see table 2); these patents are commonly under or misdiagnosed (Hopper et al, 2007).

Table 1: Gastrointestinal tract signs and symptoms of coeliac disease

- $\quad$ abdominal pain, dyspepsia

- $\quad$ severe malabsorption

- $\quad$ post-prandial abdominal distension / bloating

- $\quad$ steatorrhoea (difficult to flush pale, loose fatty stools)

- $\quad$ flatulence (often foul-smelling)

- $\quad$ vomiting

- diarrhoea

- constipation

- $\quad$ early satiety

- $\quad$ anorexia

- $\quad$ weight loss - can be severe; conversely, it is often absent and obesity does not discount a diagnosis of $C D$

- dental enamel defects, i.e. loss of enamel coating

- recurrent aphthous ulceration

Table 2: Non or extra-gastrointestinal tract signs and symptoms of coeliac disease 
- neurological presentations, such as headaches, migraines, peripheral neuropathy, epilepsy and cerebellar ataxia

- chronic fatigue

- fibromyalgia, arthralgia, arthritis

- depression and anxiety

- symptoms of anaemia, particularly iron or vitamin B12 deficiency

- osteoporosis/pathological fracture

- dermatitis herpetiformis (itchy blistering rash and trunk and limbs)

- amenorrhoea; subfertility in both females and males

- growth failure in children, pubertal delay

- vitamin D deficiency

- increased risk of recurrent miscarriage; low-birth weight children; increased infant mortality

- otherwise unexplained alopecia

Patients with MS present and experience a diverse range of neurological symptoms that manifest the damage to the different parts of the central nervous system (see figure); for some patients unremitting stealthy pathology is invariably associated with steady disease progression and disability (Gelfand, 2014).

Table: Common presenting signs \& symptoms of MS

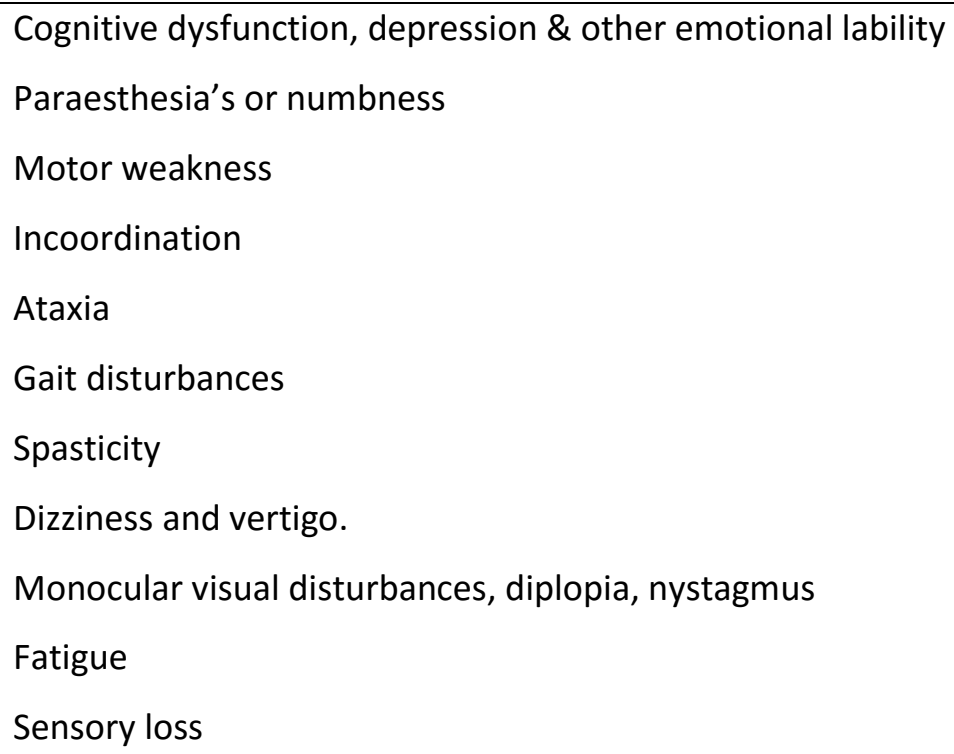


Neuropathic pain

Urinary urgency or retention

Sexual dysfunction

Heat intolerance

Lhermitte's phenomenon

\section{Neurological manifestations of coeliac disease}

Almost half of all patients with CD manifest extra-gastrointestinal signs and symptoms; just over a fifth experienced neurological or neuropsychiatric indicators of disease, including cognitive impairment, peripheral neuropathy, ataxia, headache, depression, decreased cerebellar volume, and seizures (Briani et al, 2008; Sapone et al, 2012; Currie et al, 2012; Shen et al, 2012; Dimitrova et al, 2013). Silent or subclinical neurological involvement has also been described, where it is postulated prolonged exposure to gluten in susceptible patients promoted subtle changes to brain morphology, such as decreased cortical mater volumes and changes to the caudate nucleus, which plays a crucial role in memory and learning (Bilgic et al, 2013).

The presentation of clinical neurological signs, morphology and disease trajectory associated with CD that resemble and can be misconstrued as those concomitant with MS has been reported, resulting in years of ineffective, unnecessary and costly treatment (Finsterer and Leutmezer, 2014). Equally, in patients with MS, it has been suggested gluten sensitivity should be considered in those who present with gastrointestinal indicators of disease (Azizi et al, 2014), and in those patients presenting with ataxia, or atypical MRI findings, or non-response to treatment, even in the absence of gastrointestinal symptoms (Salvatore et al, 2014).

Cognitive impairment, which may manifest as problems with attention, new learning and memory, and reduced information processing speed, is a common feature of MS with a marked negative impact upon functional status and quality of life (Mitolo et al, 2015). In adult patients with CD, cognitive impairment presenting as amnesia, acalculia, confusion and personality changes has been reported (Hu et al, 2006). More recently, milder and more subtle cognitive impairments have been reported and colloquially termed 'brain fog', and manifest as problems with attentiveness and concentration, disorientation, temporary loss of mental alertness, perception and creativity (Lichtwark et al, 2014). The authors suggested that the effect would be similar to recovery from jetlag over a $24 \mathrm{~h}$-period and clearly had implications on health and safety. 
The exact cause of cognitive impairment remains elusive. Lichtwark et al (2014) postulated several reasons. Firstly, that $\mathrm{CD}$ is associated with malabsorption, which might be associated with micronutrient deficiencies, especially those that are known to contribute to cognitive decline such as vitamin D or iron deficiency; however, there was no evidence of such a correlation. Secondly, elevated levels of circulating inflammatory cytokines act upon central signalling receptors or activate neurones, affecting mood, behaviour and cognition. Finally, using data from animal models, Lichtwark et al (2014) further speculated that gluten may have a direct chemical effect reducing the concentration of tryptophan, a precursor to serotonin; affecting opioid peptides; or changing the gut microbiota, the microorganisms that inhabit the human body; all of which may exert an influence on human brain function. As with some of the more severe symptoms, brain fog improved following adherence to a gluten free diet.

The most frequent neurological manifestations of $C D$, and other non-CD gluten sensitivities, are peripheral neuropathy and cerebellar ataxia, occurring in up to $10 \%$ of patients with $C D$ (Hadjilivassilou et al, 2010; Rashtak et al, 2012). Brannagan et al (2005) suggested that the neuropathy may be the presenting symptom of the condition and may occur without gastrointestinal tract symptoms. A recent Swedish study found individuals with coeliac disease were around 2.5 times more likely to receive a later diagnosis of neuropathy than those without celiac disease, suggesting that this significant association would merit screening for $C D$ in patients presenting with neuropathy (Thawani et al, 2015).

As with MS, the peripheral neuropathy associated with CD may be axonal or demyelinating in nature (Tengah et al, 2002), and the most commonly reported subtypes are symmetrical sensorimotor axonal peripheral neuropathy and small fibre sensory polyneuropathies, though other motor and sensorimotor neuropathies have been described (Chin \& Latov, 2005; Hadjivasiliou et al, 2006). Pain and lower limb paraesthesia are reportedly the most common frequent presentation of CD related neuropathy ; however, asymmetrical sensory symptoms and facial pains have been reported; motor weakness and autonomic symptoms are less common (Chin et al, 2003; Rezania, 2010). Luostarinen et al (2003) found evidence of higher heat pain and touch thresholds in patients with coeliac disease further suggesting that peripheral nerve fibres, even small unmyelinated fibres, are impaired. Adoption of and adherence to a gluten free diet is generally associated with an improvement in symptoms; however, Luostarinen et al (2003) found that patients with CD whose disease was in remission had nevertheless an increased risk of neuropathy, which they considered may have been due to prolonged diagnostic delay. 
Ataxia is a frequent complication of MS (Malik et al, 2014) and CD (Hu et al, 2006; Pennisi et al, 2014), and the most common neurological manifestation of gluten sensitivity (Hadjivassiliou et al, 1998).

The ataxic presentation associated with gluten include problems with gait, upper and lower limb coordination, balance and movement; dysarthria and fine-finger movements; oculomotor, sensory or bladder dysfunction; dysphonia and pyramidal signs (Sander et al, 2003; Hadjivassiliou et al, 2003; Nikpour, 2012; Hadjivassiliou et al, 2015).

The evidence for gluten ataxia has been described as 'overwhelming' and could be previously characterized by the evidence of involvement of immune system mediation by via positive antigliadin antibodies (as seen in CD), and anti-Purkinje neuron antibodies (which may share antigenic determinants, or epitopes, similar to gluten), with ensuing cerebellar inflammation and atrophy (Hadjivassiliou et al, 2004). The use of more specific and sensitive testing for auto-antibodies not normally found in the blood but present in patients with $C D$, such as anti-tissue transglutaminase (tTG-2) testing now takes place. TTG is an enzyme involved in crosslinking or connecting certain proteins in various body tissues including the cells of the gut, which is released during the inflammatory response. It is postulated it acts as a deamidating enzyme, degrading and covalently bonding to gliadin peptides, which can enhance the immunostimulatory effect and is thought to be a significant phase in the pathogenesis of coeliac disease (diSabatino et al, 2012).

It has been noted that adherence to a strict gluten-free diet sees an improvement in ataxic symptoms (Hadjivassiliou et al, 2006); however, prolonged exposure to gluten appears to cause permanent and irreparable nerve damage suggesting the need for prompt recognition and treatment to prevent progression (Volta \& deGiorgio, 2010; Lauret \& Rodrigo, 2013). The evidence for a gluten free diet in patients with MS is inconclusive, with papers suggesting there was no definitive evidence that such a diet conferred a benefit to the management of MS (Hewson et al, 1984; Schmitz et al, 2015), whilst Rodrigo et al (2014) suggested the preliminary findings of their recent randomised controlled trial had found that a gluten free diet improved the clinical and physical status of patients and seemingly exerted a neuroprotective effect.

A lack of awareness of coeliac disease is associated with delays to diagnosis, which in turn is associated with adverse effects on the quality of life for the patient as well as an augmented risk of long term complications. Though there is no compelling evidence for endorsing routine screening for $C D$ in patients with $M S$, nurses do need to have cognisance of the complex picture of two common 
diseases that have similar symptom presentation and experience of MS in order to provide appropriate assessment, advice, care and management for patients.

Box 1: Numbers of help, advice, support and information

\section{Coeliac UK}

Coeliac UK work on behalf of those with coeliac disease, providing support for everyday living and advancing understanding of the condition through research.

https://www.coeliac.org.uk/home/

Telephone helpline 08453052060

Coeliac UK Office: 3rd Floor, Apollo Centre, Desborough Road, High Wycombe, Buckinghamshire, HP11 2QW

MS?

Multiple Sclerosis Trust

Spirella Building

Bridge Road

Letchworth Garden City

Hertfordshire

SG6 4ET

Tel: 01462476700

Free Phone Information Service: 08000323839

Fax: 01462476710

email: info@mstrust.org.uk

\section{Coeliac disease and Multiple Sclerosis references}

Batur-Caglayan, HZ; Irkec, C; Yildirim-Capraz, I et al (2013) A case of multiple sclerosis and celiac disease. Care reports in Neurological Medicine dx.doi.org/10.1155/2013/576921 
Barrow, R \& Craig, D (2009) Genetics of multiple sclerosis Barrow Quarterly 24(2): 13-16

Bilgic, B; Aygun, D; Arslan, AB et al (2013) Silent neurological involvement in biopsy-defined coeliac patients Neurological Sciences 34(2): 2199-2204

Bomprezzi, \& Craig, D (2009) genetics of multiple sclerosis Barrow Quarterly 24(2): 13-16

Boullerne, Al; Skias, D; Hartman, EM et al (2015)A Single-Nucleotide Polymorphism in SerineThreonine Kinase 11, the Gene Encoding Liver Kinase B1, Is a Risk Factor for Multiple Sclerosis American Society of Neurochemistry Neuro. January -February: 1-13

Brannagan, TH; Hays, AP; Chin, SS et al (2005) Small-Fiber Neuropathy/Neuronopathy Associated With Celiac Disease: Skin Biopsy Findings. Archives of Neurology \& Psychiatry. 62(10):1574-1578

Briani C, Zara G, Alaedini A, et al (2008) Neurological complications of celiac disease and autoimmune mechanisms: a prospective study Journal of Neuroimmunology 195(1-2):171-5.

Compston, A \& Cole, A (2008) Multiple Sclerosis Lancet 372(9648): 1502-1517

Currie,S; Hadjivassiliou, M; Clark, MJR et al (2012) Should we be 'nervous' about coeliac disease? Brain abnormalities in patients with coeliac disease referred for neurological opinion Journal of Neurology, Neurosurgery \& Psychiatry 83:1216-1221

Chin RL, Sander HW, Brannagan TH, et al (2003) Celiac neuropathy. Neurology. 60(10):1581-1585. 
Chin, RL \& Latov, N (2005) Peripheral Neuropathy and Celiac Disease. Current Treatment Options in Neurology. 7(1):43-48.

Dimitrova AK, Ungaro RC, Lebwohl B, et al (2013) Prevalence of migraine in patients with celiac disease and inflammatory bowel disease. Headache. 53(2):344-55

Di Sabatino, A \& Corazza, GR (2009) Coeliac disease Lancet 373: 1480-93

Di Sabatino A; Vanoli A; Giuffrida P et al (2012) The function of tissue transglutaminase in celiac disease. Autoimmunity Reviews 11(10):746-53

Ferro, M; Franciotta, D; Riccardi, T et al (2008) A case of multiple sclerosis with atypical onset associated with auto-immune hepatitis and silent coeliac disease Neurological Sciences 1: 29-31

Finsterer, J \& Leutmezer, F (2014) Celiac disease with cerebral and peripheral involvement mimicking multiple sclerosis Journal of Medicine and Life 7(3): 440-444

Gelfand, JM (2014) Multiple sclerosis: diagnosis, differential diagnosis and clinical presentation IN Handbook of Clinical Neurology 122: 269-290

Goddard, CJR \& Gillett, HR (2006) Complications of coeliac disease: are all patients at risk? Postgraduate Medical Journal 82: 705-712

Hadjivassiliou, M; Grunewald, R; Sharrack, B et al (2003) Gluten ataxia in perspective: epidemiology, genetic susceptibility and clinical characteristics Brain 123: 685-691 
Hadjivassiliou, M; Williamson, CA; Woodroofe, N (2004) The immunology of gluten sensitivity: beyond the gut. Trends in Immunology 25(11):578-582

Hadjivassiliou, M; Grunewald, RA; Kandler, RH et al (2006) Neuropathy associated with gluten sensitivity Journal of Neurology, Neurosurgery \& Psychiatry 77:1262-1266

Hadjivassiliou M; Kandler RH; Chattopadhyay AK et al (2006b) Dietary treatment of gluten neuropathy. Muscle and Nerve. 34:762-766.

Hadjivassiliou, M ; Sanders, DS \& Grünewald, RA et al (2010) Gluten sensitivity: from gut to brain. Lancet Neurology 9: 318-330

Hadjivassiliou M; Sanders D; Aeschlimann D (2015) Gluten-Related Disorders: Gluten Ataxia. Digestive Disorders 33:264-268

Hall, A \& Yates, C (2010) Organ Specific auto-immunity IN Immunology OUP. Oxford Hewson, DS (1984) Is there a role for gluten-free diets in multiple sclerosis? Human Nutrition Applied Nutrition, 38: 417-420

Hopper, AD, Hadjivassiliou, M, Butt, S et al (2007) Adult coeliac disease. BMJ. 335: 558-562

Hu, WT; Murray, JA; Greenaway, MC et al (2006) Cognitive impairment and celiac disease. Archives of Neurology 63: 1440-1446 
Husby, S; Koletzko, S; Korponay-Szabo, IR et al (2012) European Society for Pediatric Gastroenterology, Hepatology, and Nutrition Guidelines for the Diagnosis of Coeliac Disease Journal of Paediatric Gastroenterology \& Nutrition 54: $136-160$

Jiang, I-L, Zhang, B-L; Liu, Y-S (2009) Is adult celiac disease really uncommon in Chinese? Journal of Zhejiang University 10(3):168-171

Jones, R \& Sleet, S (2009) Easily missed? Coeliac disease. BMJ 338: 3058

Karussis, D (2014) The diagnosis of multiple sclerosis and the various related demyelinating syndromes: A critical review. Journal of Autoimmunity 48-49 (2014) 134-142

Kuma, V; Rajadhyaaksha, M; Wortsman, J (2001) Celiac disease-associated endocrinopathies. Clinical and diagnostic immunology 8(4): 678-685

Lauret, E and Rodrigo, L (2013) Celiac Disease and Autoimmune-Associated Conditions. Biomed Research International. doi: 10.1155/2013/127589

Lebenthal, E; Shteyer, E and Branski, D (2008) The changing clinical presentation of celiac disease Frontiers in Celiac Disease. Paediatric Adolescent Medicine. 12:18-22

Lichtwark, IT; Newnham, ED; Robinson, SR et al (2014) Cognitive impairment in coeliac disease improves on a gluten-free diet and correlates with histological and serological indices of disease severity Alimentary Pharmacology \& Therapeutics. 40(2):160-70

Ludvigsson, JF; Card, TR; Kakuninen, K et al (2015) Screening for celiac disease in the general population and in high risk groups. United European Gastroenterology Journal 3(2): 106-120 
Murray, JA; Watson, T; Clearman, B et al (2004) Effect of a gluten-free diet on gastrointestinal symptoms in celiac disease American Journal of Clinical Nutrition 79(4): 669-673

Nicoletti A; Patti F; Lo Fermo, S et al (2008) Frequency of celiac disease is not increased among multiple sclerosis patients. Multiple Sclerosis Journal 14:698-700

Nikpour, S (2012) Neurological manifestations, diagnosis, and treatment of celiac disease: A comprehensive review. Iranian Journal of Neurology Iranian Journal of Neurology 11(2): 59-64

Mackenzie IS; Morant SV; Bloomfield GA et al (2013) Journal of Neurology Neurosurgery and Psychiatry doi:10.1136/jnnp-2013-305450

Malik, O; Donnelly, A \& Barnett, M (2014) Fast facts: Multiple sclerosis Health Press. Oxford.

Milo, R \& Miller, A (2014) Revised diagnostic criteria for multiple sclerosis Autoimmunity Reviews $13(4-5): 518-524$

Mitolo, M; Veneeri, A; Wilkinson, ID et al (2015) Cognitive rehabilitation in MS: A systematic review. Journal of Neurological Sciences 354:1-9

Pagliari, D; Urgesi, R; Frosali, S et al (2015) The interaction among microbiota, immunity, and genetic and dietary factors is the condicio sine qua non celiac disease can develop. Journal of Immunology Research: 2015:123653.doi: 10.1155/2015/123653. Epub 2015 May 18. 
Pennisi, G; Lanza, G; Giuffrida, S et al (2014) Excitability of the motor cortex in de novo patients with celiac disease. PLOS ONE 9(7):e102790.doi:10.1371/journal.pone.0102790

Rashtak, S; Rashtak, S; Snyder, MR et al (2011) Serology of celiac disease in gluten sensitive ataxia or neuropathy: role of deamidated gliadin antibody. Journal of Neuroimmunology 230: 130-134

Reilly NR, Green PH. (2012) Epidemiology and clinical presentations of celiac disease. Seminars in Immunopathology. 34(4):473-8.

Rezania, K (2010) Celiac neuropathy. Impact. University of Chicago. Chicago

Rodrigo, L; Hernandez-Lahoz, C; Fuentes, D et al (2011) Prevalence of celiac disease in multiple sclerosis BMC Neurology 11(31): 1-7

Rodrigo, L; Hernandez-Lahoz, C; Fuentes, D et al (2014) Randomised clinical trial comparing the efficacy of a gluten free diet versus regular diet in a series of relapsing remitting multiple sclerosis patients. International Journal of Neurology and Neurotherapy 1(2): 1-6

Rubio-Tapia A, Ludvigsson JF, Brantner TL, et al (2012) The prevalence of celiac disease in the United States. American Journal of Gastroenterology 107:1538-44

Salvatore, S; Tozza, A \& Nespoli, L (2014) Celiac disease in Multiple Sclerosis: A controversial issue. International Journal of Neurology \& Neurotherapy 1:006, 1-3

Sander, HW; Magda, P; Chin, RL et al (2003) Cerebellar ataxia and coeliac disease: case report Lancet 362(9395): 1548 
Sapone, A; Bai, JC; Ciacci, C et al (2012) Spectrum of gluten related disorders: consensus on new nomenclature and classification. BMC Medicine 10(13):1-12

Schmitz, K; Barthelmes, J; Stolz, L et al (2015) "Disease modifying nutricals" for multiple sclerosis Pharmacology \& Therapeutics 148: 85-113

Shaygannejad, V; Ghasemi; Mirmohammedasedhi, M (2013) Multiple sclerosis or neurological manifestations of celiac disease. Advanced Biomedical Research 2(1): 1-3

Shen TC, Lebwohl B, Verma H, et al (2012) Peripheral neuropathic symptoms in celiac disease and inflammatory bowel disease. Journal of Clinical Neuromuscular Disease. 13(3):137-45

Simpson Jr, S; Blizzard, L; Otahal, P et al (2011) Latitude is significantly associated with the prevalence of multiple sclerosis: a meta-analysis. Journal of Neurology Neurosurgery and Psychiatry 82: $1132-1141$

Tengah, DSNAP; Wills, AJ \& Holmes, GKT (2002) Neurological complications of coeliac disease. Postgraduate Medical Journal 78: 393-398

Thawani, SP; Brannagan, TH; Lebwohl, B et al (2015) Risk of neuropathy among 28232 patients with biopsy verified celiac disease JAMA Neurology 72(7): 806-811

Tjon, JM-L; van Bergen, J; Koning, F et al (2010) Celiac disease: how complicated can it get? Immunogenetics 62: 641-651 
Ward-Abel, N \& Burgoyne, T (2008) The importance of the immune response in multiple sclerosis, part 1: Pathophysiology British journal of Neuroscience Nursing 4(5): 212-217

Viera, C, Matos, M, Quarema, T et al (2011) What do Brazilian Pediatricians know about Celiac disease? Digestive Diseases and Sciences. 56: 799-804

Visser EM; Wilde, K; Wilson, JF et al (2012) A new prevalence study of multiple sclerosis in Orkney, Shetland and Aberdeen city Journal of Neurology, Neurosurgery and Psychiatry 2012;83(7):719-724.

Visser, J, Rozing, J, Sapone, A et al (2009) Tight junctions, intestinal permeability and autoimmunity celiac disease and type 1 diabetes paradigms. Annals of the New York Academy of Science 1165: 195205

Volta, U \& deGiorgio, R (2010) Gluten sensitivity: an emerging issue behind neurological impairment. Lancet Neurology 9:233-235

Williams, SAL, Heather, N, Beattie, RM (2010) Coeliac disease Paediatrics and Child 20(10); 457-461

Zarkadas, M; Cranney, A; Case, S et al (2006) The impact of a gluten free diet on adults with coeliac disease: results of a national survey. Journal of Human Nutrition and Dietetics 19: 41-49

Zipser, RD, Farid, M, Basich, D et al (2005) Physician awareness of coeliac disease: a need for further education. Journal of General Internal Medicine 20: 644-646 
